\title{
TOROIDAL ALGEBRAIC GROUPS ${ }^{1}$
}

\section{MAXWELL ROSENLICHT}

Many of the more striking elementary properties of abelian varieties generalize to other kinds of algebraic groups, e.g. tori, i.e. direct products of multiplicative groups $G_{m}$, and in fact to extensions of tori by abelian varieties. This seems to have been more or less known for some time, but explicit statements are hard to find, a lack we attempt to remedy here. We may mention specifically a recent paper of S. Arima [1], reading between the lines of which might lead one in this direction. In what follows, the better-known structural facts on algebraic groups are used freely; most of these are in [2] and [4], while the relevant facts on abelian varieties can be found in either [7] or [3].

PROPOSITION. The following properties of a connected algebraic group $G$ are equivalent:

(1) The maximal connected linear algebraic subgroup of $G$ is a torus.

(2) $G$ contains no algebraic subgroup that is isomorphic to the additive group $G_{a}$.

(3) For any connected algebraic subgroup $H$ of $G$, the points of $H$ of finite order prime to the field characteristic are dense in $H$.

Proof. The equivalence of (1) and (2) is a consequence of the fact that a connected linear algebraic group is a torus if and only if it contains no $G_{a}$ (cf. [2]). If (1) or (2) holds, clearly any connected algebraic subgroup of $G$ is of the same type. If $T$ is the maximal connected linear subgroup of $G$ then the points of finite order prime to the field characteristic of the abelian variety $G / T$ are dense in $G / T$, and similarly for the torus $T$. Thus we get (3). Conversely, (3) trivially gives (2).

A group $G$ such as in the proposition we shall call toroidal. Such a group is necessarily commutative and has only a finite number of elements of any given finite order $n$. As a matter of fact, if $n$ is prime to the field characteristic the elements of order dividing $n$ form a subgroup of $G$ that is the direct product of a certain number of cyclic groups of order $n$, this number being $\operatorname{dim} T+2 \operatorname{dim} G / T$ if $T$ is the

Received by the editors December 1, 1960 .

1 This research was partially supported by the United States Air Force through the Air Force Office of Scientific Research of the Air Research and Development Command, under Contract No. AF 49(638)-603. Reproduction in whole or in part is permitted for any purpose of the United States Government. 
maximal torus of $G$; this is immediate from the corresponding facts for tori and abelian varieties, and is the basic tool of Arima [1], who takes $n$ to be a variable power of a fixed prime $l$ ( $\neq$ characteristic) and so gets $l$-adic matricial representations for homomorphisms between toroidal groups.

COROLLARY. If the toroidal group $G$ is a normal algebraic subgroup of the connected algebraic group $\Gamma$, then $G$ is contained in the center of $\Gamma$. Any algebraic group that is isogenous to a toroidal group, or is a connected algebraic subgroup of a toroidal group, or a homomorphic image of a toroidal group, or an extension of one toroidal group by another, is also toroidal.

The first fact follows from consideration of points of finite order, the rest from the corresponding facts for abelian varieties and tori.

LеммA. Let $V$ be a variety, $A$ an abelian variety. Then the rational maps $\phi: V \rightarrow A$ form, modulo constant maps, a free abelian group with a finite number of generators.

That there is no torsion in this group is clear, so finite generation is all that must be shown. This may be done by replacing $V$ by its albanese variety, noting that any rational map from an abelian variety $B$ into $A$ is (modulo a translation) a homomorphism, and using the knowledge that $\operatorname{Hom}(B, A)$ is a finite $Z$-module. However we wish to make two remarks: First, the theory of the albanese variety is not necessary for the proof, for $V$ may be replaced by a generic curve on it (cf. [3, pp. 41-42]), hence by a jacobian variety. Second, a very easy proof may be given in the classical case. For this, note that since a rational map $\phi: V \rightarrow A$ is defined at each simple point of $V$, we may restrict our attention to such maps $\phi$ that are everywhere defined. It then suffices to show that if $\phi$ induces the zero map on the homology group $H_{1}(V, Z)$ then $\phi$ is constant. In this case, uniformizing $A$, we are reduced to showing that any bounded holomorphic function on $V$ is constant, which reduces to the easy case $\operatorname{dim} V=1$.

It is easy to see that for each of the following Theorems $1-3$, the given property actually characterizes toroidal groups in the class of all connected algebraic groups.

Theorem 1. Let $V$ be a variety, $G$ a toroidal algebraic group. Then the everywhere defined rational maps $\phi: V \rightarrow G$ form, modulo constant maps, a free abelian group with a finite number of generators.

The lemma reduces us to the case where $G$ is a torus, hence to the case $G=G_{m}$. But this latter case is known $[5$, lemma to Proposition 
3]. (Outline of proof for the case $G=G_{m}$ : We may take $V$ to be normal and affine, hence the affine part of a normal projective variety $\bar{V}$. But an everywhere defined nowhere zero function on $V$ is determined, up to a constant factor, by its orders on the various components of $\bar{V}-V$.)

Theorem 2. Let $V, W$ be varieties, $G$ a toroidal algebraic group, $\phi: V \times W \rightarrow G$ an everywhere defined rational map. Then there exist everywhere defined rational maps $\phi_{1}: V \rightarrow G, \phi_{2}: W \rightarrow G$ such that, for any $(v, w) \in V \times W, \phi(v, w)=\phi_{1}(v)+\phi_{2}(w)$.

Letting $\psi_{1}, \cdots, \psi_{r}: V \rightarrow G$ be a set of generators, modulo constants, for the group of all everywhere defined rational maps $\psi: V \rightarrow G$, letting $k$ be a field of definition for $V, W, G, \phi, \psi_{1}, \cdots, \psi_{r}$, and letting $P$ be a generic point of $W$ over $k$, there exist integers $n_{1}, \cdots, n_{r}$ such that

$$
\phi(v, P)=n_{1} \psi_{1}(v)+\cdots+n_{r} \psi_{r}(v)+f_{P},
$$

where $f_{P} \in G$ is a constant point, rational over $k(P)$. All we have to do now is let $\phi_{2}: W \rightarrow G$ be the rational map, defined over $k$, such that $\phi_{2}(P)=f_{P}$.

THEOREM 3. Let $\phi: \Gamma \rightarrow G$ be an everywhere defined rational map from a connected algebraic group $\Gamma$ into a toroidal algebraic group $G$, with $\phi(e)=0$. Then $\phi$ is a homomorphism.

An algebraic group of the form $G=A T$, where $A, T$ are algebraic subgroups of $G, A$ being abelian and $T$ a torus, is clearly toroidal, but not all toroidal groups are of this form. For an example, start with a nonsingular curve $C$ and $r>1$ distinct points $P_{1}, \cdots, P_{r}$ of $C$. Then there is a projective model $C_{\mathrm{m}}$ of $C$ which is nonsingular except for one point, at which the local ring consists precisely of all functions on $C$ which are defined and take on equal values at $P_{1}, \cdots, P_{r}$ ( $m$ denotes the divisor $\left.P_{1}+\cdots+P_{r}\right)$. The generalized jacobian $J_{\mathfrak{m}}$ of $C_{\mathfrak{m}}$ is then toroidal, its maximal torus having dimension $r-1$. If $J_{\mathfrak{m}}$ were of the form $A T$ it would possess a nontrivial homomorphism into a torus, hence into $G_{m}$, and the restriction of such a function to the canonical image of $C$ would be a nonconstant rational function on $C$ the carrier of whose divisor is contained in $|\mathfrak{m}|=P_{1} \cup \ldots \cup P_{r}$, and we know that such a function cannot exist for $C$ of genus $>0$ and $P_{1}, \cdots, P_{r}$ chosen properly. However Arima has shown [1] that any toroidal group that is defined over a finite field is actually of the form $A T$; in fact he has the following slightly stronger result, for which we offer a different proof. 
THEOREM 4. If $G$ is a connected algebraic group that is defined over a finite field and $A$ is its maximal abelian subvariety and $L$ its maximal connected linear algebraic subgroup, then $G=A L$.

The smallest normal algebraic subgroup $D$ of $G$ such that $G / D$ is linear is, according to the general structure theory [4], connected and commutative, contains only a finite number of elements of any given finite order (in particular, order equal to the field characteristic), and is such that $G=D L, L$ being as above. Since $D$ is toroidal, we may assume to begin with that $G$ is toroidal. $G$ is generated by its curves through 0 that are defined over finite fields, hence may be assumed to be generated by one such curve, so that, according to [6], it is a homomorphic image of a generalized jacobian variety. As a matter of fact, since $G$ is toroidal this generalized jacobian may be taken to be of the type $J_{\mathfrak{m}}$ of the previous paragraph; this follows from $[6$, p. 84$]$, or by noting that since the unipotent part of this generalized jacobian must map into 0 in $G$ the generalized jacobian may be replaced by a quotient, which is now another generalized jacobian, of the type $J_{\mathfrak{m}}$. Hence we may suppose that $G=J_{\mathfrak{m}}$, where $\mathfrak{m}=P_{1}+\cdots+P_{r}$ is a divisor on a curve $C, C$ and each $P_{i}$ being defined over a finite field. For each $i=1, \cdots, r-1$, the divisor $P_{i}-P_{r}$ has an image on the ordinary jacobian variety of $C$ that is contained in the subgroup of points that are rational over a certain finite field, so that $P_{i}-P_{r}$ is of finite order in the divisor class group, i.e. there is a rational function $f_{i}$ on $C$ such that $f_{i}\left(P_{i}\right)=0, f_{i}\left(P_{r}\right)=\infty$ and $f_{i}$ is elsewhere finite and nonzero. The rational map $\psi: C \rightarrow\left(G_{m}\right)^{r-1}$ that is given by $\psi(p)=\left(f_{1}(p), \cdots, f_{r-1}(p)\right)$ is defined at each point of $C-|\mathfrak{m}|$. Using the universal mapping property of generalized jacobians, as above, we get a rational homomorphism $\tau: J_{\mathfrak{m}} \rightarrow\left(G_{m}\right)^{r-1}$ such that $\psi(p)=\tau(\phi(p))+\alpha, \phi: C \rightarrow J_{\mathfrak{m}}$ being the canonical map and $\alpha$ being a constant. Altering each $f_{i}$ by a nonzero constant factor, we can assume $\alpha=0$, so $\psi=\tau \phi$. $\tau$ must be surjective, for otherwise there would be integers $n_{1}, \cdots, n_{r-1}$, not all zero, such that $f_{1}^{n_{1}} \cdots f_{r-1}^{n_{r-1}}$ $=1$, which is impossible. Noting that $J_{\mathfrak{m}}$ is an extension of a torus of dimension $r-1$ by the ordinary jacobian of $C$, we see that the component of the identity of the kernel of $\tau$ is an abelian variety of the correct dimension.

In this paper we have refrained from considering problems involving fields of definition. There are a number of such problems of interest, for example the theory of the $K / k$-image and $K / k$-trace of a toroidal group defined over an extension field $K$ of $k$ (oral remark of S. Lang), but the tools for handling these are at hand. 


\section{REFERENCES}

1. S. Arima, Commutative group varieties, J. Math. Soc. Japan vol. 12 (1960) pp. 227-237.

2. A. Borel, Groupes linéaires algêbriques, Ann. of Math. vol. 64(1956) pp. 20-82.

3. S. Lang, Abelian varieties, New York, Interscience, 1959.

4. M. Rosenlicht, Some basic theorems on algebraic groups, Amer. J. Math. vol. 78 (1956) pp. 401-443.

5. - Some rationality questions on algebraic groups, Ann. Mat. Pura Appl. vol. 43 (1957) pp. 25-50.

6. - A universal mapping property of generalized jacobian varieties, Ann. of Math. vol. 66 (1957) pp. 80-88.

7. A. Weil, Variêtés abéliennes et courbes algébriques, Paris, Hermann, 1948.

University of California, Berkeley

\section{EXTENDING CHARACTERS ON SEMIGROUPS}

\section{KENNETH A. ROSS ${ }^{1}$}

W. W. Comfort has proved [1, Theorem 4.2] a theorem on approximating certain semicharacters on commutative semigroups. He used the structure theory established in [2] and expressed doubt as to the necessity of one of his hypotheses, namely core $S(\chi) \neq \Lambda$. His result suggested the following theorem, which tells us when a character on a subsemigroup of a commutative semigroup $G$ can be extended to a character on $G$. Because of its technical nature we will not state Comfort's theorem but we will state as a corollary to our theorem a result which implies his theorem directly (with the hypothesis core $S(\chi) \neq \Lambda$ dropped).

A bounded complex-valued function $\psi$ on a semigroup $G$ is called a semicharacter of $G$ if $\psi(x) \neq 0$ for some $x \in G$ and $\psi(x y)=\psi(x) \psi(y)$ for all $x, y \in G$. A character $\psi$ is a semicharacter for which $|\psi(x)|=1$ for all $x \in G$. We note that it follows from the theorem in [3] that any character can be extended to a semicharacter.

TheOREm. Let $G$ be a commutative semigroup and let $S \subseteq G$ be a subsemigroup. A character $\psi$ on $S$ can be extended to a character on $G$ if and only if $\psi$ satisfies:

$$
a, b \in S, x \in G, \text { and } a x=b x \text { imply } \psi(a)=\psi(b) .
$$

Received by the editors June 20, 1960 and, in revised form, October 27, 1960.

${ }^{1}$ Supported by a National Science Foundation pre-doctoral fellowship. 